\section{Military Technical College Kobry El-Kobbah, Cairo, Egypt}

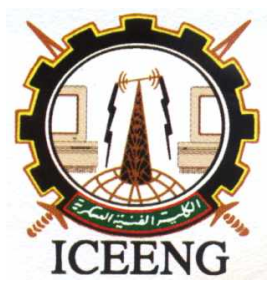

\section{$6^{\text {th }}$ International Conference on Electrical Engineering ICEENG 2008}

\title{
Segmentation of iranian bank checks
}

\author{
By
}
S.Alirezaee *
M.Allahiari *
F.Askari*
A.Alijamat *]

\section{Abstract:}

This paper aims to provide a segmentation algorithm for the Iranian bank checks. The central idea is based on the morphological analysis and connected component concept. The proposed algorithm uses the connected component properties to segment unconstrained Iranian bank checks and prepares those for OCR application. To evaluate the performance of the algorithm, it has been tested on a set of 240 Iranian bank checks which collected from 6 types of Iranian bank checks (40 samples for each type). The algorithm has a good success on document restoration and segmentation. Numerical results indicate that the proposed algorithm has $96.582 \%$ accuracy on segmentation of 5 fields of the Iranian bank checks.

\section{Keywords:}

Document analysis, segmentation, connected component, mathematical morphology

* Islamic Azad University, Abhar Branch, Abhar, Iran Alirezaee@znu.ac.ir 


\section{Introduction:}

Bank check segmentation and recognition is a well-known topic in the document analysis [1]. Through this research, we aim to provide an algorithm for segmentation of the Iranian bank checks. Some of difficulties in these documents consist of: different template, size and contents and unconstrained handwritings Fig.(1).The central concept of the proposed algorithm is based on the mathematical morphology and the connected component analysis.

This paper has been organized as follows. The second section describes a typical document restoration and segmentation block. In this section, the essential document analysis blocks and the selected strategies are discussed with details. The third section presents the proposed segmentation algorithm. The numerical results are presented in the fourth section. Finally, the conclusions will be discussed.

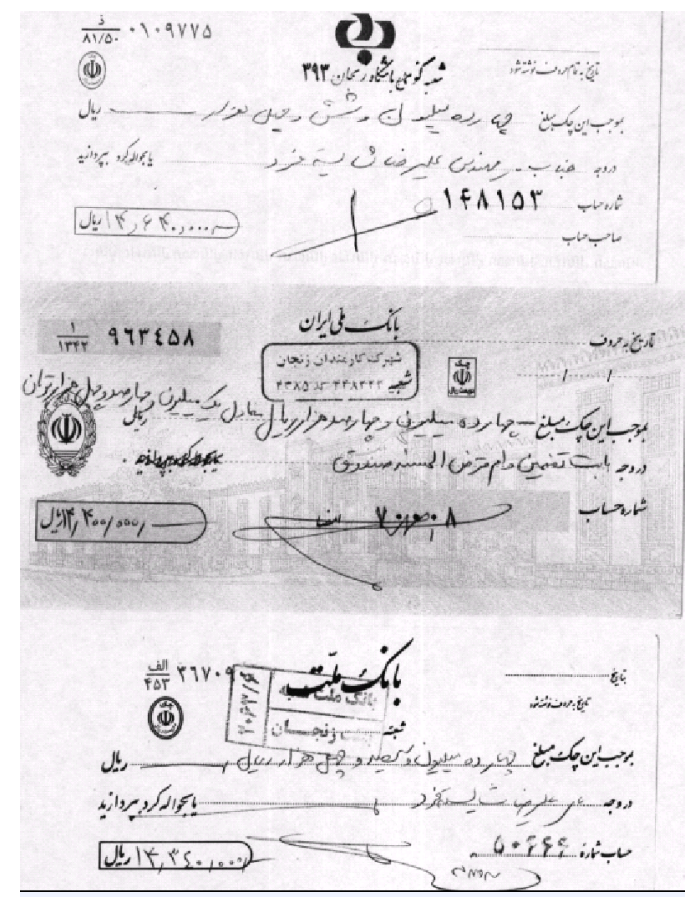

Fig.1. Samples of the Iranian bank checks

\section{Segmentation:}

The final goals in off-line handwriting recognition are character and word recognition. It is necessary to perform several document restoration and segmentation operations prior to recognizing text in scanned documents. Some of the common operations performed prior to recognition are [1,2]: Thresholding, the task of converting a gray-scale image into a binary black-white image[3]; noise removal, the extraction of the foreground textual matter by removing, textured background, salt and pepper noise and interfering 
strokes; line segmentation, the separation of individual lines of text; word segmentation, the isolation of textual words, and character segmentation, the isolation of individual characters. The central concept of the proposed algorithm is based on the mathematical morphology and the connected component analysis which will be discussed with details.

\section{1. Morphological smoothing}

They are two fundamental morphological operators, erosion and dilation. The erosion of A by $B$ is defined as [4,5]:

$$
A \otimes B=\left\{z(B)_{z} \subset A\right\}
$$

When $\mathrm{A}$ is eroded by $\mathrm{B}$, the latter is called a structuring element. Eroding an image by a structuring element $B$ has the effect of 'shrinking' the image in a manner determined by B. Larger B causes more erosion effects on A.

The dilation of $\mathrm{A}$ by $\mathrm{B}$, denoted $A \oplus B$, is defined as:

$$
A \oplus B=\left\{z\left|(B)_{z}\right| \quad A \neq \Phi\right\}
$$

Dilation expands the image based on the structure element characteristics.

Based on these two fundamental operators, many other functions have been defined which opening and closing are the most important. Opening generally smoothes the contour of an object, breaks narrow isthmuses, and eliminates these protrusions. Closing also tend to smooth section of contours but, as opposed to opening, it generally fuses narrow breaks and long thin gulfs, eliminates small holes and fills gaps in the contour the opening of the set $\mathrm{A}$ by structuring element $\mathrm{B}$, denoted $A C B$, is defined as:

$$
A \mathrm{O} B=(A \otimes B) \oplus B
$$

and similarly, the closing of set $\mathrm{A}$ by structuring element $\mathrm{B}$, denoted $A * B$, is defined as:

$$
A * B=(A \oplus B) \otimes B
$$

Morphological smoothing operator is an opening followed by a closing. The net results of these two operators can remove (or attenuate) both bright and dark artifacts and noises. On the other hand, these operators are capable to connect undesired discontinuities caused in the previous stages and as well as smooth the inner and outer contours.

\subsection{Morphological Connected component}

Let $\mathrm{Y}$ represent a connected component contained in a set $\mathrm{A}$ and assume that a point $\mathrm{P}$ of $Y$ is known. Then the following iterative expression yields all the elements of $Y$ [10]: $X_{k}=\left(X_{k-1} \oplus B\right) \mid A$

where $X_{0}=p$, and $\mathrm{B}$ is a suitable structuring element. If $X_{k}=X_{k-1}$, the algorithm has converged and we let $Y=X_{k}$. We have used the simple structure element in this work. 


\section{Proposed Segmentation Algorithm:}

Due to the mentioned difficulties of the Iranian bank checks, some serious preprocessing and image restoration algorithm should apply before OCR process. The proposed document analysis algorithm has been presented in Fig.2. In the first step, a global thresholding is applied on the scanned page. Then the morphological smoothing is used for noise removal. In the next step, initial baseline candidates are extracted from the horizontal histogram maximas. Then a clustering k_Means [6] algorithm will merge the baseline candidates and the true baselines i.e. $\left\{l_{1}, l_{2}, \ldots, l_{M}\right\}$ are yielded. As can be seen in the next section, the applied procedure has a good performance for baseline detection. The other task of this block is line width estimation. We have applied the same procedure on the horizontal histogram minimas to find the best separation area for the successive lines. The result will be the best candidate for between line gaps. The average distance between these gaps (with illuminating the first and last lines) is considered as the line width and is labeled 2.4. In the next block an initial line candidate is extracted as:

$$
\begin{aligned}
& \text { Line }_{i}=\text { Input } \_ \text {page }\left(l_{i}-\alpha . \Delta: l_{i}+\alpha . \Delta,:\right) \\
& \quad i=1,2, \ldots, M, \alpha \in[1,1.2] \\
& \text { which, }
\end{aligned}
$$

Input _ page : The original document page (not the thresholded image)

$l_{i}: i^{\prime}$ th baseline position

$\alpha$ : The amount of line overlapping

Line $_{i}: i^{\prime}$ th extracted text line 


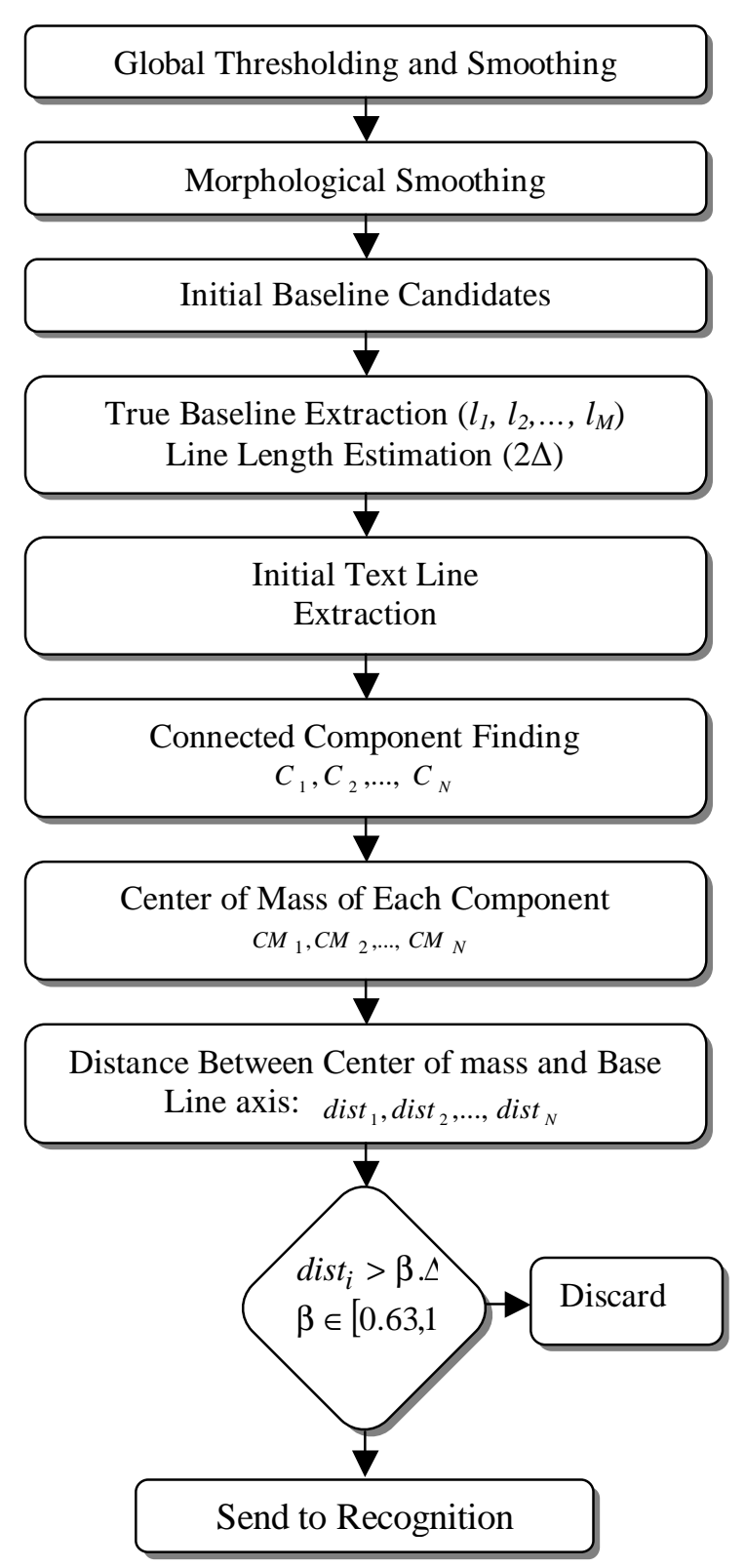

Fig.2. Block diagram of the proposed segmentation algorithm

As can be seen in (6), for more precision, line is extracted from the original image. Therefore we should have the other thresholding and noise removing process. In the next stage, morphological connected component detection is used for detecting the line components [5]. These undesired components have several sources such as overlapping part of neighbor lines, noise and thresholding effects. We have made a decision rule for discarding undesired components. Proposed strategy uses the distance between component center of mass $\left(\right.$ dist $\left._{i}\right)$ and baseline and as well as the component size $\left(\operatorname{surf}_{i}\right)$, i.e. :

$$
\text { dist }_{i}=\left\|C M_{i}-l_{i}\right\|
$$


and

$$
\operatorname{surf}_{i}=\sum C_{i}
$$

The next block compares this distance with the following thresholds:

If dist $_{i}>\beta . \Delta \rightarrow$ Discard the component

If dist $_{i}<\beta . \Delta \rightarrow$ Sent to recognition phase

Which,

$$
\beta \in[.63,1]
$$

\section{Numerical Results:}

A set of 240 check samples has been scanned from different types of Iranian bank checks which have been collected from 6 types of Iranian bank checks (40 samples for each type). There are 5 fields in the Iranian bank check structures which listed in the table 1 .

Table 1. Different fields of the Iranian bank checks

\begin{tabular}{|c|c|}
\hline Field Name & Location Type \\
\hline Date & constrained \\
\hline Signature & unconstrained \\
\hline $\begin{array}{c}\text { Amount in } \\
\text { number }\end{array}$ & constrained \\
\hline Amount in letter & constrained \\
\hline Order & constrained \\
\hline
\end{tabular}

The proposed segmentation algorithm has been tested on the database. Table. 2 presents the results over the database.

Table 2. Different fields of the Iranian bank checks

\begin{tabular}{|c|c|c|}
\hline Field Name & $\begin{array}{c}\text { Number of correct field segmentation(on 240 } \\
\text { check images) }\end{array}$ & $\begin{array}{c}\text { Recognition } \\
\text { rate }\end{array}$ \\
\hline Date & 240 & $100 \%$ \\
\hline Signature & 24 & $90 \%$ \\
\hline $\begin{array}{c}\text { Amount in } \\
\text { number }\end{array}$ & 239 & $99.58 \%$ \\
\hline Amount in letter & 230 & $95.83 \%$ \\
\hline Order & 234 & $97.5 \%$ \\
\hline Total & 240 & $96.582 \%$ \\
\hline
\end{tabular}




\section{Conclusions:}

In this paper a segmentation algorithm for the Iranian bank checks was proposed. The central idea is based on the morphological analysis and connected component concept. The proposed algorithm applied the connected component properties to segment unconstrained Iranian bank checks and prepares those for OCR application. To evaluate the performance of the algorithm, it has been tested on a set of 240 Iranian bank checks which collected from 6 types of Iranian bank checks (40 samples for each type). Numerical results indicate that the proposed algorithm has $96.582 \%$ accuracy on segmentation of 5 fields of the Iranian bank checks.

\section{Acknowledgement:}

The authors would like to thank the Islamic Azad University of Abhar for supporting this research.

\section{References:}

[1] A.K.Jain, B.Yu, Document Representation and Its Application to Page Decomposition, IEEE Trans. on Pattern Analysis and Machine Intelligence, VOL.20, NO.3, pp. 294-308, MARCH 1998.

[2] A. Zlatopolsky, "Automated Document Segmentation," Pattern Recognition Letters, vol. 15, pp. 699-704, 1994.

[3] P.K.Sahoo, S.Soltani, A.K.C.Wong, and Y.C.Chen, "Asurvey of thresholding techniques", Computer Vision, Graphics, and Image Processing, Vol.41, pp.233-260, 1988.

[4] J. Ha, R. Haralick, and I. Phillips, "Document Page Decomposi-tion by the Bounding-Box Projection Technique," Proc. Third Int'l Conf. Document Analysis and Recognition, pp. 1,119-1,122, Montreal, 1995.

[5] M.A.Lopez, and R.Thurimella, On computing connected components of line segments, IEEE Trans. on Computers, VOL. 44, NO. 4, April 1995.

[6] A.Likas, N.Valassis, J.J.Verbeek, The global k_means algorithm, Pattern Recognition, Vol. 36, 451-461, May 2003. 